\title{
Editorial: Physical Layer Security and Wireless Access Control (QSHINE 2017)
}

\author{
Lei Wang ${ }^{1} \cdot$ Jenhui Chen ${ }^{2} \cdot$ Yang Peng ${ }^{3} \cdot$ Lei Zhang ${ }^{4}$ \\ Published online: 16 April 2019 \\ (C) Springer Science+Business Media, LLC, part of Springer Nature 2019
}

\section{Editorial:}

Wi-Fi has become a predominant means of communication. However, the problem of unauthorized access to Wi-Fi network has become increasingly severe. Physical-layer security is emerging as a promising approach for supporting new and existing security services. Using properties of physical-layer communication channels in security mechanisms can potentially enhance conventional security services and assist in mitigating threats that challenge the capabilities of conventional cryptographic mechanisms, such as spoofing attack, relay attacks, ad-hoc key establishment and key-less secure communication. However, significant challenges, including security evaluation and guarantees, remain to be solved to realize practical physical-layer security mechanisms.

The aim of this Special Issue is to publish the state-of-the-art research results demonstrating both theoretical and practical approaches to physical-layer security. Through a peer-review process, we have accepted 13 papers from a total of 25 papers submitted to this special issue, which means an acceptance rate of $52 \%$.

The first paper entitled "Security Performance Analysis of Underlay Cognitive Networks with Helpful Jammer Under Interference from Primary Transmitter" y employs a helpful jammer to secure information transmission of a secondary transmitter. Then, its efficacy through secrecy outage probability under practical considerations consisting of exponentially distributed interference from primary transmitter, peak transmission power limitation, interference power limitation,

Lei Wang

lei.wang@ieee.org

\footnotetext{
Dalian University of Technology, Dalian, China

Chang Gung University, Taoyuan, Taiwan

University of Washington Bothell, Bothell, WA, USA

Tianjing University, Tianjin, China
}

and Rayleigh fading channels are analytically assessed. Finally, results are provided to illustrate significant security performance improvement thanks to exploiting the jammer while considerable security performance degradation due to interference from primary transmitter.

The second paper entitled "An Auxiliary Antenna Based Inter-User Interference Mitigation Approach in Full-Duplex Wireless Networks" proposes an IUI suppression scheme that based on the auxiliary antenna at the downlink user. The single-cell simulation results show that the average achievable rate of the proposed IUI suppression scheme significantly outperforms the existing schemes especially when the cell radius is small and can nearly approach the performance of the ideal FD case with IUI perfectly suppressed.

In the third paper entitled "Outage Performance Analysis of Energy Harvesting Wireless Sensor Networks for NOMA Transmissions" investigates radio frequency energy harvesting in wireless sensor networks using non-orthogonal multiple access uplink transmission regarding a probable secrecy outage during the transmission between sensor nodes and base station in the presence of eavesdroppers.

The fourth paper focused "Protecting Source Location Privacy in a Clustered Wireless Sensor Networks against Local Eavesdroppers", where the author proposes three source privacy protection schemes, which are based on clustering methodology, to protect contextual privacy.

In the fifth paper entitled "Anomaly Detection in UASN Localization Based on Time Series Analysis and Fuzzy Logic", the authors propose a novel anomaly detection system for underwater acoustic sensor network localization. The results indicate that anomaly detection systems offer an acceptable accuracy with high true positive rate and F-Score.

In the sixth paper entitled "Enhancing Efficient Link Performance in ZigBee Under Cross-Technology Interference", the authors propose a novel adaptive packet delivery (APD) algorithm that enables ZigBee links to achieve enhanced performance under the presence of heavy Wi-Fi traffic. 
In the seventh paper entitled "A Novel Semi-fragile Digital Watermarking Scheme for Scrambled Image Authentication and Restoration", the authors design and implement a semifragile watermark based on cat transformation, mostly used to locate tamper and recover for the transformed image and plain-image.

The rapid development of the Unmanned Aerial Vehicle (UAV) brings much convenience to our life. However, security and privacy problems caused by UAVs are gradually exposed. The eighth paper entitled "Security and Privacy Issues of UAV: A Survey" analyzes UAV safety from three aspects, including sensors, communications and multi-UAVs.

Software-Defined Networking (SDN) has been proposed as a promising way for its centralized network control and management. The ninth paper entitled "Joint Optimization of Latency Monitoring and Traffic Scheduling in Software Defined Heterogeneous Networks" designs a monitoring loop of link latency by using both LLDP and Echo probing modules. The tenth paper entitled "WECAN: An Efficient WestEast Control Associated Network for Large-Scale SDN Systems" proposes an efficient West-East Control Associated Network for enabling communication between different SDN entities.

It is a hot topic to protect the data of the wireless network stored in the cloud. In the eleventh paper entitle "ConfusingKeyword Based Secure Search over Encrypted Cloud Data", the authors present a privacy protection scheme based on confusing keyword.

The twelfth paper entitled "Applying Improved Convolutional Neural Network in Image Classification" presents an integrated optimization method of simulated annealing (SA) and Gaussian convolution based on Convolutional Neural Network.

The last paper entitled "Performance Specifications for the Roll-Off Factor and Filter Order for Filtered Multitone Modulation in the Maritime VHF Data Exchange System", where the authors systematically analyzed the effects of the filter parameters on communication performance in FMT modulation. Based on the simulated and real-world results, they recommend performance specifications for the roll-off factor and filter order for FMT modulation in the VDES.

Acknowledgements The guest editors are thankful to our reviewers for their effort in reviewing the manuscripts. We also thank the Edit-in-Chief, Dr. Imrich Chlamtac for his supportive guidance during the entire process.

Publisher's Note Springer Nature remains neutral with regard to jurisdictional claims in published maps and institutional affiliations.

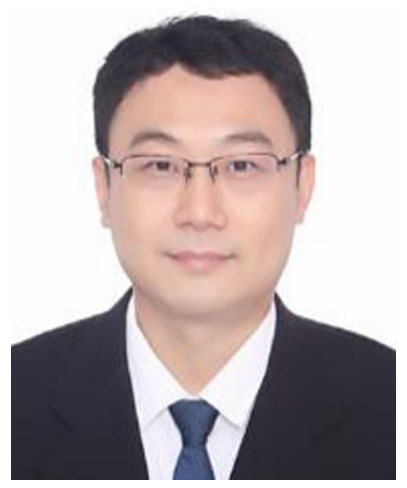

Lei Wang (M'01) received the B.S., M.S., and Ph.D. degrees from Tianjin University, China, in 1995, 1998, and 2001, respectively. He was a Member of Technical Staff with Bell Labs Research China from 2001 to 2004, a Senior Researcher with Samsung, South Korea, from 2004 to 2006, a Research Scientist with Seoul National University from 2006to 2007, and a Research Associate with Washington State University, Vancouver, WA, USA from 2007 to 2008. He is currently a Full Professor with the School of Software, Dalian University of Technology, China. He has published over 100 papers and his papers have received about 2000 citations. His research interests involve wireless ad hoc network, sensor network, social network, and network security.

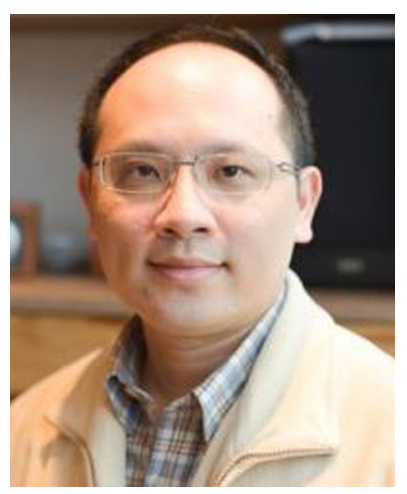

Jenhui Chen (S'99-M'03SM'14) received the B.S. and Ph.D. degrees in Computer Science and Information Engineering (CSIE) from Tamkang University, Taipei, Taiwan, R.O.C. in July 1998 and January 2003, respective1y. Dr. Chen joined the Department of CSIE, School of Electrical and Computer Engineering, College of Engineering, Chang Gung University since 2003. He is currently a full professor and the chairman of the Department of CSIE, Chang Gung University. He is the head of the graduate studies section, academic affairs office, Chang Gung University. Dr. Chen is a professor of Center for Artificial Intelligence in Medicine, Chang Gung Memorial Hospital. His main research interests include design, analysis, and implementation of communication protocols, wireless networks, artificial intelligence (AI), human-like intelligence (HI), deep learning, data science, and network-on-chip (NoC) for multi-core and multi-processor system-on-chip (MPSoC) design. Dr. Chen is a senior member of IEEE. He is currently a senior editor of Cogent Engineering and an editor of The Scientific World Journal and Advances in Computer Engineering. Dr. Chen served as the technical program committee (TPC) member of IEEE Globecom 2016-2019, IEEE VTC 2018, IEEE ICC 2015, IEEE ICCC 2018-2019, IEEE ICCCN 20142018, IEEE 5G World Forum 2018-2019, and ACM CCIOT 2019. He also served as a reviewer for many famous academic journals which are organized by $\mathrm{ACM}$, Elsevier, IEEE, and Springer. 


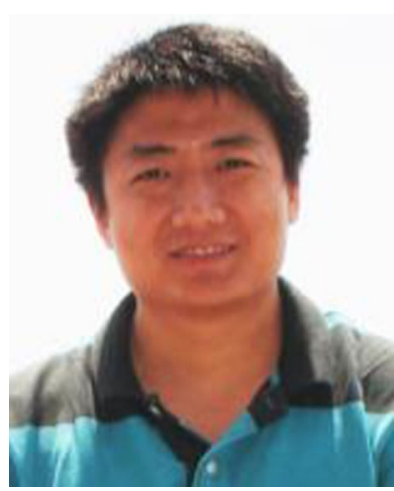

Yang Peng received his $\mathrm{PhD}$ degree in Computer Science from Iowa State University, USA, in 2014. He is currently an Assistant Professor in the Division of Computing and Software Systems, University of Washington Bothell, WA, USA. His research interests include Internet of things, wireless sensor networks and cyber-physical systems.

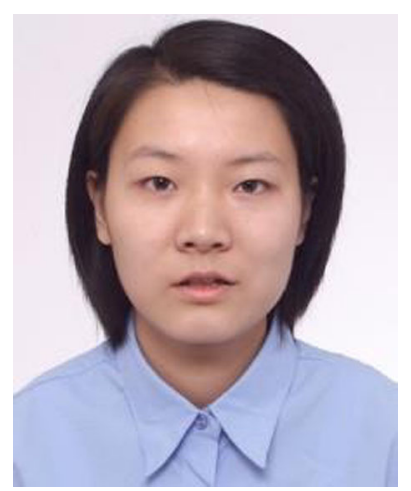

Lei Zhang received the Ph.D. degree in computer science from Auburn University, Auburn, AL, USA, in 2008. She was an Assistant Professor with the Computer Science Department, Frostburg State University, Frostburg, MD, USA, from 2008 to 2011. She is currently an Associate Professor with the School of Computer Science and Technology, Tianjin University, Tianjin, China. Her current research interests include computer networks, wireless communications, distributed algorithms, and network security. 